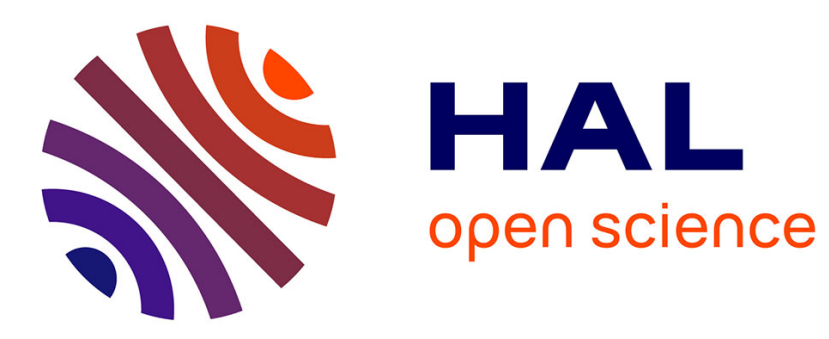

\title{
Regularity of prime ideals
}

Giulio Caviglia, Marc Chardin, Jason Mccullough, Irena Peeva, Matteo

Varbaro

\section{To cite this version:}

Giulio Caviglia, Marc Chardin, Jason Mccullough, Irena Peeva, Matteo Varbaro. Regularity of prime ideals. Mathematische Zeitschrift, 2019, 291 (1-2), pp.421-435. 10.1007/s00209-018-2089-y . hal03004657

\section{HAL Id: hal-03004657 https://hal.science/hal-03004657}

Submitted on 27 Nov 2020

HAL is a multi-disciplinary open access archive for the deposit and dissemination of scientific research documents, whether they are published or not. The documents may come from teaching and research institutions in France or abroad, or from public or private research centers.
L'archive ouverte pluridisciplinaire HAL, est destinée au dépôt et à la diffusion de documents scientifiques de niveau recherche, publiés ou non, émanant des établissements d'enseignement et de recherche français ou étrangers, des laboratoires publics ou privés. 


\title{
REGULARITY OF PRIME IDEALS
}

\author{
GIULIO CAVIGLIA, MARC CHARDIN, JASON MCCULLOUGH, IRENA PEEVA, \\ AND MATTEO VARBARO
}

\begin{abstract}
We answer several natural questions which arise from the recent paper [MP] of McCullough and Peeva providing counterexamples to the Eisenbud-Goto Regularity Conjecture. We give counterexamples using Rees algebras, and also construct counterexamples that do not rely on the Mayr-Meyer construction. Furthermore, examples of prime ideals for which the difference between the maximal degree of a minimal generator and the maximal degree of a minimal first syzygy can be made arbitrarily large are given. Using a result of Ananyan-Hochster we show that there exists an upper bound on regularity of prime ideals in terms of the multiplicity alone.
\end{abstract}

\section{Introduction}

Regularity is a numerical invariant that measures the complexity of the structure of homogeneous ideals in a polynomial ring. It has been studied in Algebraic Geometry and Commutative Algebra; see the expository paper [Ch]. We consider a standard graded polynomial ring $U=k\left[z_{1}, \ldots, z_{p}\right]$ over a field $k$, where all variables have degree one. Let $L$ be a homogeneous ideal in the ring $U$, and let $\beta_{i j}(L)=\operatorname{dim}_{k} \operatorname{Tor}_{i}^{U}(L, k)_{j}$ be its graded Betti numbers. The (Castelnuovo-Mumford) regularity of $L$ is

$$
\operatorname{reg}(L)=\max \left\{j \mid \beta_{i, i+j}(L) \neq 0\right\} .
$$

Alternatively, regularity can be defined using local cohomology. Papers of Bayer-Mumford, Bayer-Stillman, and Koh, give examples of families of ideals attaining doubly exponential regularity. In contrast, Bertram-Ein-Lazarsfeld, Chardin-Ulrich, and Mumford have proved that there are nice bounds on the regularity of the ideals of smooth (or nearly smooth) projective varieties; see the expository paper [Ch2]. As discussed in the influential paper [BM] by Bayer and Mumford (1993), the biggest missing link between the general case and the smooth case is to obtain a decent bound on the regularity of all prime ideals (the ideals that define irreducible projective varieties). The long standing Eisenbud-Goto Regularity Conjecture predicts an elegant linear bound, in terms of the degree of the variety:

2010 Mathematics Subject Classification. Primary: 13D02.

Key words and phrases. Syzygies, Free Resolutions, Castelnuovo-Mumford Regularity.

Caviglia is partially supported by NSA MSP grant H98230-15-1-0004. Peeva is partially supported by NSF grant DMS-1702125. 
The Regularity Conjecture 1.1. (Eisenbud-Goto [EG], 1984) Suppose that the field $k$ is algebraically closed. If $L \subset\left(z_{1}, \ldots, z_{p}\right)^{2}$ is a homogeneous prime ideal in $U$, then

$$
\operatorname{reg}(L) \leq \operatorname{deg}(U / L)-\operatorname{codim}(L)+1
$$

where $\operatorname{deg}(U / L)$ is the multiplicity of $U / L$ (also called the degree of $U / L$, or the degree of $X=\operatorname{Proj}(U / L))$, and $\operatorname{codim}(L)$ is the codimension (also called height) of $L$.

The conjecture is proved for curves by Gruson-Lazarsfeld-Peskine, for smooth surfaces by Lazarsfeld and Pinkham, for most smooth 3-folds by Ran and Kwak, if $U / L$ is CohenMacaulay by Eisenbud-Goto, and in many other special cases.

Recently, McCullough and Peeva [MP] introduced two new techniques and used them to provide many counterexamples to the Eisenbud-Goto Regularity Conjecture. In this note we answer some natural questions which arise from the paper [MP].

The counterexamples in $[\mathrm{MP}]$ come from Rees-like algebras, which were introduced in [MP, Section 3]. Rees-like algebras, unlike the usual Rees algebras, have well-structured defining equations and minimal free resolutions. The properties of Rees algebras are of high interest and can be quite intricate (see for example [Hu], [KPU]). Several mathematicians have asked us if the defining ideals of Rees algebras contain counterexamples as well or whether the Regularity Conjecture holds for them. In Sections 3 and 4 we provide counterexamples using Rees algebras. In the latter section we study standard graded Rees algebras that arise as Rees algebras of ideals generated in one degree.

The main theorem in [MP] shows that the regularity of prime ideals is not bounded by any polynomial function of the multiplicity. It is natural to ask if there exists a bound on regularity in terms of the multiplicity alone. Such a bound does not exist for primary ideals (Example 5.4). However, we prove in Section 5 that the recent work of Ananyan-Hochster $[\mathrm{AH}]$ (who solved Stillman's Conjecture) implies the existence of the desired bound for prime ideals. Other proofs of Stillman's Conjecture are given in [ESS, DLL].

In the counterexamples in $[\mathrm{MP}]$ the multiplicity is smaller than the maximal degree of a minimal generator of a prime ideal. One may wonder whether there are prime ideals for which the difference between the maximal degree of a minimal generator and the maximal degree of a minimal (first) syzygy can be made arbitrarily large. In Section 6 we show that such prime ideals exist. We obtain them by starting with Ullery's designer ideals (which are not prime) [Ul] and applying to them the method by McCullough-Peeva in order to get prime ideals.

In Section 7 we construct a family of three-generated ideals whose regularity grows faster than the product of the degrees of the generators. To our knowledge, this is the only known such family other than those based on the Mayr-Meyer construction. Applying the construction in $[\mathrm{MP}]$ we use this family to construct an infinite family of counterexamples to the Eisenbud-Goto Regularity Conjecture that do not rely on the Mayr-Meyer construction. 


\section{Multiplicity of prime ideals}

Throughout this section, we consider a polynomial ring $W=k\left[w_{1}, \ldots, w_{p}\right]$ over an arbitrary field $k$ and positively graded with $\operatorname{deg}\left(w_{i}\right) \in \mathbb{N}$ for every $i$. Suppose $c_{i}:=\operatorname{deg}\left(w_{i}\right)>1$ for $i \leq q$ and $\operatorname{deg}\left(w_{i}\right)=1$ for $i>q$ (for some $q \leq p$ ).

A function $Q: \mathbb{Z} \longrightarrow \mathbb{Q}$ is a quasipolynomial (over $\mathbb{Q}$ ) of degree $r$ if

$$
Q(n)=a_{r}(n) n^{r}+a_{r-1}(n) n^{r-1}+\cdots+a_{1}(n) n+a_{0}(n),
$$

where $a_{i}: \mathbb{Z} \longrightarrow \mathbb{Q}$ is a periodic function for each $i=0, \ldots, r$ and $a_{r} \neq 0$. A natural number $v$ is called a period of $Q$ if

$$
a_{i}(n+v)=a_{i}(n) \text { for all } n \in \mathbb{Z} \text { and for all } i=0, \ldots, r .
$$

Let $M$ be a homogeneous ideal in the polynomial ring $W$. The Hilbert function $h_{W / M}$ : $\mathbb{Z}_{\geq 0} \longrightarrow \mathbb{Z}_{\geq 0}$ of $W / M$ is $h_{W / M}(n)=\operatorname{dim}_{k}(W / M)_{n}$. It is often studied via the Hilbert series

$$
\operatorname{Hilb}_{W / M}(u)=\sum_{n \geq 0} u^{n} \operatorname{dim}_{k}(W / M)_{n} .
$$

By a theorem of Hilbert-Serre, there is a quasipolynomial $Q(n)$ of degree $\operatorname{dim}(W / M)-1$ and period $\operatorname{lcm}\left(c_{1}, \ldots, c_{p}\right)$ such that

$$
h_{W / M}(n)=Q(n) \text { for } n \gg 0 .
$$

For a proof, see for example [BI].

Set $d:=\operatorname{gcd}\left(c_{1}, \ldots, c_{p}\right)$, and observe that $Q(d j+t)=0$ for $0<t<d$.

Proposition 2.1. If $M$ is a prime ideal, then $a_{r}(d j)$ is a constant (independent of the parameter $j$ ), which we denote $a_{r}$.

Proof. We may easily reduce to the case $d=1$ by dividing the degrees of the variables by their greatest common divisor.

Assume the opposite. Set $a(n)=a_{r}(n)$. Let $m$ and $m+s$ be two different integers for which the Hilbert function agrees with the quasipolynomial $Q$ and such that $a(m)>$ $a(m+s)$. Since $\operatorname{gcd}\left(c_{1}, \ldots, c_{p}\right)=1$, there exist $\ell_{i} \in \mathbb{Z}$ such that $s=\sum_{1}^{q} \ell_{i} c_{i}$. Hence, $m+s=m+\sum_{1}^{q} \ell_{i} c_{i}$. Adding a large positive multiple of $b:=\operatorname{lcm}\left(c_{1}, \ldots, c_{p}\right)$ to the righthand-side, we get

$$
a(m+s)=a\left(m+\sum_{i=1}^{q} \ell_{i} c_{i}+v b\right)=a\left(m+\sum_{i=1}^{q} \ell_{i}^{\prime} c_{i}\right)
$$

where each $\ell_{i}^{\prime}$ is positive. Foir each $i$, as $w_{i}$ is a non-zerodivisor, we have an inclusion $w_{i}(W / M)_{j} \subseteq(W / M)_{j+c_{i}}$ and thus $\operatorname{dim}_{k}(W / M)_{j} \leq \operatorname{dim}_{k}(W / M)_{j+c_{i}}$ for every $j \geq 0$. Hence,

$$
a(m+s)=a\left(m+\sum_{i=1}^{q} \ell_{i}^{\prime} c_{i}\right) \geq a(m),
$$

which is a contradiction. 
If $M$ is a prime ideal in $W$, then we call

$$
e_{\text {Hilb }}(M):=r ! a_{r}
$$

the Hilbert multiplicity of $S / M$ or of $M$, and also denote it by $\operatorname{deg}(M)$ or $e_{\mathrm{Hilb}}(W / M)$.

On the other hand, recall the construction of the Euler polynomial:

Notation 2.2. Fix a finite graded complex $\mathbf{V}$ of finitely generated $W$-free modules and with $V_{i}=0$ for $i<0$. We may write $V_{i}=\oplus_{j \in \mathbf{Z}} W(-j)^{b_{i j}}$. The Euler polynomial of $\mathbf{V}$ is

$$
\mathrm{E}_{\mathbf{V}}=\sum_{i \geq 0} \sum_{j \in \mathbf{Z}}(-1)^{i} b_{i j} u^{j}
$$

Let $N$ be a graded finitely generated $W$-module, and let $\mathbf{V}$ be a finite graded free resolution of $N$. Since every graded free resolution of $N$ is isomorphic to the direct sum of the minimal graded free resolution and a trivial complex, it follows that the Euler polynomial does not depend on the choice of the resolution, so we call it the Euler polynomial of $N$. We factor out a maximal possible power of $1-u$ and write

$$
\mathrm{E}_{\mathbf{V}}=(1-u)^{c} h_{\mathbf{V}}(u),
$$

where $h_{\mathbf{V}}(1) \neq 0$.

We set $N=W / M$ and in the notation above, we call

$$
e_{\text {Euler }}(M):=h_{\mathbf{V}}(1)
$$

the Euler multiplicity of $S / M$ or of $M$, and also denote it by $e_{E u l e r}(W / M)$.

A prime ideal $M$ is called non-degenerate if $M \subset\left(w_{1}, \ldots, w_{p}\right)^{2}$.

Theorem 2.3. If $M$ is a non-degenerate homogeneous prime ideal in $W$, then

$$
e_{\text {Euler }}(M)=e_{\text {Hilb }}(M) \prod_{i=1}^{p} \operatorname{deg}\left(w_{i}\right) .
$$

The proof uses the technique of step-by-step homogenization introduced in [MP]. Theorem 2.3 is an immediate corollary of Theorem 2.5.

The following result from $[\mathrm{MP}]$ describes the step-by-step homogenization technique:

Step-by-step Homogenization Theorem 2.4. [MP] Let $M$ be a homogeneous nondegenerate prime ideal, and let $\mathcal{K}$ be a minimal set of homogeneous generators of $M$. Consider the homogenous map (of degree 0)

$$
\begin{aligned}
\nu: W=k\left[w_{1}, \ldots, w_{p}\right] & \longrightarrow W^{\prime}:=k\left[w_{1}, \ldots, w_{p}, v_{1}, \ldots, v_{q}\right] \\
w_{i} & \longmapsto w_{i} v_{i}^{\operatorname{deg}_{W}\left(w_{i}\right)-1} \quad \text { for } 1 \leq i \leq q,
\end{aligned}
$$

where $v_{1}, \ldots, v_{q}$ are new variables and $W^{\prime}$ is standard graded. The ideal $M^{\prime} \subset W^{\prime}$ generated by the elements of $\nu(\mathcal{K})$ is a homogeneous non-degenerate prime ideal in $W^{\prime}$. Furthermore, the graded Betti numbers of $W^{\prime} / M^{\prime}$ over $W^{\prime}$ are the same as those of $W / M$ over $W$. 
We say that $M^{\prime}$ is obtained from $M$ by step-by-step homogenization or by relabeling. The relation between the multiplicities of $M$ and $M^{\prime}$ is given in the next result:

Theorem 2.5. In the notation and under the assumptions of Theorem 2.4 we have

$$
\begin{aligned}
e_{\text {Euler }}\left(M^{\prime}\right) & =e_{\text {Hilb }}\left(M^{\prime}\right) \\
e_{\text {Euler }}\left(M^{\prime}\right) & =e_{\text {Euler }}(M) \\
e_{\text {Hilb }}\left(M^{\prime}\right) & =e_{\text {Hilb }}(M) \prod_{i=1}^{p} \operatorname{deg}\left(w_{i}\right) .
\end{aligned}
$$

Proof. The first equality holds because $M^{\prime}$ is a homogeneous ideal in a standard graded ring. The second equality holds because step-by-step homogenization preserves the graded Betti numbers by Theorem 2.4. We will prove the third equality.

We have the same Euler polynomial $\mathrm{E}(u):=\mathrm{E}_{\mathbf{M}}=\mathrm{E}_{\mathbf{M}^{\prime}}$ by Theorem 2.4. We get the Hilbert series

$$
\begin{aligned}
\operatorname{Hilb}_{W / M}(u) & =\frac{\mathrm{E}(u)}{(1-u)^{p-q} \prod_{i=1}^{q}\left(1-u^{\operatorname{deg}\left(w_{i}\right)}\right)} \\
\operatorname{Hilb}_{W^{\prime} / M^{\prime}}(u) & =\frac{\mathrm{E}(u)}{(1-u)^{p+q}} .
\end{aligned}
$$

Therefore,

$$
\begin{aligned}
\operatorname{Hilb}_{W^{\prime} / M^{\prime}}(u) & =\frac{\prod_{i=1}^{q}\left(1-u^{\operatorname{deg}\left(w_{i}\right)}\right)}{(1-u)^{2 q}} \operatorname{Hilb}_{W / M}(u) \\
& =\frac{\prod_{i=1}^{q}\left(1+u+\cdots+u^{\operatorname{deg}\left(w_{i}\right)-1}\right)}{(1-u)^{q}} \operatorname{Hilb}_{W / M}(u) .
\end{aligned}
$$

Note that the factor in front of $\operatorname{Hilb}_{W / M}(u)$ is a series with positive coefficients. Apply Lemma 2.6.

If $S / M$ has Hilbert multiplicity $a$, we say that its Hilbert series has it too.

Lemma 2.6. Let $h$ be a Hilbert function given by a quasipolynomial $Q(n)$ of degree $r$ and with constant leading coefficient $a:=r ! a_{r}$. Let $g$ be the Hilbert series of $h$.

(1) The Hilbert multiplicity of the Hilbert series $\frac{g}{1-u}$ is the same.

(2) The Hilbert multiplicity of the Hilbert series $\left(1+u+\cdots+u^{b}\right) g$ is $(b+1) a$.

Proof. We have $g=\sum_{n \geq 0} Q(n) u^{n}$.

(1) Since

$$
\left(1+u+u^{2}+\cdots\right) g=\sum_{n \geq 0} u^{n}(Q(0)+Q(1)+\cdots+Q(n))
$$


the quasipolynomial for the considered Hilbert series is

$$
\begin{aligned}
Q(n)+\cdots+Q(0)= & a_{r}\left(n^{r}+(n-1)^{r}+\cdots+1\right) \\
& +a_{r-1}\left(n^{r-1}+(n-1)^{r-1}+\cdots+1\right) \\
& +\cdots \\
= & \frac{a_{r}}{r+1} n^{r+1}+\text { terms of lower degree. }
\end{aligned}
$$

Thus the Hilbert multiplicity is $(r+1) ! \frac{a_{r}}{r+1}=r ! a_{r}$.

(2) Since

$$
\left(1+u+\cdots+u^{b}\right) g=\sum_{n \geq 0} u^{n}(Q(n)+\cdots+Q(n-b))
$$

the quasipolynomial for the considered Hilbert series is

$$
\begin{aligned}
Q(n)+\cdots+Q(n-b)= & a_{r}\left(n^{r}+(n-1)^{r}+\cdots+(n-b)^{r}\right) \\
& +a_{r-1}\left(n^{r-1}+(n-1)^{r-1}+\cdots+(n-b)^{r-1}\right) \\
& +\cdots \\
= & a_{r}(b+1) n^{r}+\text { terms of lower degree. }
\end{aligned}
$$

\section{Multiplicities of Rees algebras and Rees-like algebras}

In this section we provide counterexamples to the Eisenbud-Goto Regularity Conjecture [EG] using Rees algebras.

Notation 3.1. We follow the notation in $[\mathrm{MP}]$. Consider the polynomial ring

$$
S=k\left[x_{1}, \ldots, x_{n}\right]
$$

over a field $k$ with a standard grading defined by $\operatorname{deg}\left(x_{i}\right)=1$ for every $i$. Let $I$ be a homogeneous ideal minimally generated by forms $f_{1}, \ldots, f_{m}$ of degrees $a_{1}, \ldots, a_{m}$, where $m \geq 2$.

Theorem 3.2. Consider the Rees algebra $S[I t]$ and the Rees-like algebra $S\left[I t, t^{2}\right]$. We have:

$$
\begin{aligned}
e_{\text {Hilb }}(S[I t]) & \leq e_{\text {Hilb }}\left(S\left[I t, t^{2}\right]\right)=1 \\
e_{\text {Euler }}(S[I t]) & \leq \frac{1}{2} e_{\text {Euler }}\left(S\left[I t, t^{2}\right]\right)=\prod_{i=1}^{m}\left(\operatorname{deg}\left(f_{i}\right)+1\right) .
\end{aligned}
$$

Denote by $Q$ and $L$ the defining ideals of $S\left[I t, t^{2}\right]$ and $S[I t]$ respectively. Then

$$
\operatorname{deg} L^{\prime} \leq \frac{1}{2} \operatorname{deg} Q^{\prime}
$$

where $L^{\prime}$ and $Q^{\prime}\left(Q^{\prime}\right.$ is denoted by $P$ in $\left.[\mathrm{MP}]\right)$ are the respective step-by-step homogenizations of $L$ and $Q$. 
Proof. Note that

$$
\operatorname{dim}(S[t])=\operatorname{dim}(S[I t])=\operatorname{dim}\left(S\left[I t, t^{2}\right]\right)=n+1
$$

since they are domains of the same transcendence degree. Since $S[I t] \subset S\left[I t, t^{2}\right] \subset S[t]$ we conclude

$$
0<e_{\mathrm{Hilb}}(S[I t]) \leq e_{\mathrm{Hilb}}\left(S\left[I t, t^{2}\right]\right) \leq e_{\mathrm{Hilb}}(S[t])=1 .
$$

The first and second equalities below come from Theorem 2.3:

$$
\begin{aligned}
e_{\text {Euler }}(S[I t]) & =e_{\text {Hilb }}(S[I t]) \prod_{i=1}^{m} \operatorname{deg}\left(y_{i}\right) \leq e_{\text {Hilb }}\left(S\left[I t, t^{2}\right]\right) \prod_{i=1}^{m} \operatorname{deg}\left(y_{i}\right) \\
& =\frac{1}{2} e_{\text {Euler }}\left(S\left[I t, t^{2}\right]\right)=\frac{1}{2} e_{\text {Euler }}\left(Q^{\prime}\right)=\prod_{i=1}^{m}\left(\operatorname{deg}\left(f_{i}\right)+1\right) .
\end{aligned}
$$

The factor $\frac{1}{2}$ comes from the variable $z$ which has degree 2 . The last equality holds by [MP, Theorem 1.6(2)], and the equality before holds by Theorem 2.5.

The inequality $\operatorname{deg} L^{\prime} \leq \frac{1}{2} \operatorname{deg} Q^{\prime}$ now follows from Theorem 2.5.

For a graded ideal $N$ (in a positively graded polynomial ring), we denote by $\operatorname{maxdeg}(N)$ the maximal degree of an element in a minimal system of homogeneous generators of $N$.

Theorem 3.3. For $r \in \mathbf{N}$ we consider the step-by-step homogenization $L_{r}^{\prime}$ of the defining ideal $L_{r}$ of the Rees algebra $S\left[I_{r} t\right]$, where $I_{r}$ is the Koh ideal used in Counterexample 1.8(1) in $[\mathrm{MP}]$. Then multiplicity and maxdeg of the prime ideal $L_{r}^{\prime}$ satisfy

$$
\begin{aligned}
\operatorname{deg} L_{r}^{\prime} & \leq 2 \times 3^{22 r-3} \\
\operatorname{maxdeg} L_{r}^{\prime} & \geq 2^{2^{r-1}}+1 .
\end{aligned}
$$

Thus it is a counterexample to the Regularity Conjecture (see [MP, 1.2]) for $r \geq 10$.

Proof. Note that $L_{r}$ contains all the $y$-linear minimal generators listed in [MP, (3.4)]. They are minimal generators of $L_{r}$ by [MP, Proposition 2.9] and since $L_{r}$ cannot contain any elements in which no $y_{1}, \ldots, y_{m}$ appears.

Let $P_{r}$ be the prime ideal used in Counterexample 1.8(1) in [MP]. Then

$$
\operatorname{deg} L_{r}^{\prime} \leq \frac{1}{2} \operatorname{deg} P_{r} \leq 2 \times 3^{22 r-3}
$$

by Theorem 3.2 and [MP, Counterexample 1.8(1)].

Similarly, Counterexample 1.8(2) in [MP] leads to Rees-algebra counterexamples to the Regularity Conjecture. 


\section{Standard graded Rees Algebras}

In this section, we provide a different view than Theorem 3.3 on using Rees Algebras to produce examples of large regularity. We focus on standard graded Rees Algebras, which arise as the Rees algebras of ideals generated in one degree.

First, we observe how to reduce to the case of ideals generated in one degree:

Construction 4.1. We follow the notation in $[\mathrm{MP}]$. Consider the standard graded polynomial ring $S=k\left[x_{1}, \ldots, x_{n}\right]$ over a field $k$. Let $I$ be a homogeneous ideal minimally generated by forms $f_{1}, \ldots, f_{m}$ of degrees $a_{1}, \ldots, a_{m}$, where $m \geq 2$. Set $d=\max _{i}\left\{a_{i}\right\}$. Consider a new ideal $\widetilde{I}$ generated by the forms $\left\{x^{d-a_{i}} f_{i}\right\}$ of degree $d$ in the polynomial $\operatorname{ring} \widetilde{S}=S[x]$. We bigrade $\widetilde{S}$ by $\operatorname{deg}\left(x_{i}\right)=(1,1)$ for every $i$ and $\operatorname{deg}(x)=(0,1)$. The ideal $\widetilde{I}$ is bigraded, and therefore $\widetilde{S} / \widetilde{I}$ has a bigraded minimal free resolution $\widetilde{\mathbf{U}}$ over $\widetilde{S}$. The regularity $\tilde{r}$ of $\widetilde{S} / \widetilde{I}$ (assuming standard grading) is equal to the regularity of $\widetilde{\mathbf{U}}$ with respect to the second coordinate of the bigrading. It follows that it is bigger than the regularity $r^{\prime}$ of $\widetilde{\mathbf{U}}$ with respect to the first coordinate of the bigrading since $\operatorname{deg}(x)=(0,1)$. Observe that $x-1$ is a non-zerodivisor (for degree reasons using the second coordinate of the bigrading) on $\widetilde{S} / \widetilde{I}$. Therefore, $\widetilde{\mathbf{U}} \otimes \widetilde{S} /(x-1)$ is a graded (posibly non-minimal) free resolution of $S / I$ over $S$. Hence, the regularity $r$ of $S / I$ is smaller than $r^{\prime}$. We showed that

$$
\operatorname{reg}_{S}(I) \leq \operatorname{reg}_{\widetilde{S}}(\widetilde{S} / \widetilde{I}) .
$$

In fact, we have such an inequality in every homological degree, that is,

$$
\operatorname{maxdeg}\left(\operatorname{Syz}_{i}^{S}(S / I)\right) \leq \operatorname{maxdeg}\left(\operatorname{Syz}_{i}^{\widetilde{S}}(\widetilde{S} / \widetilde{I})\right),
$$

where $\operatorname{maxdeg}(N)$ stands for the maximal degree in a system of minimal homogeneous generators of a graded finitely generated module $N$.

Now, we consider the Rees Algebra $R:=\widetilde{S}[\widetilde{I} t]$ as a standard graded ring. Its prime graded (with respect to the standard grading) defining ideal $T$ satisfies

$$
\operatorname{maxdeg}(T) \geq \operatorname{maxdeg}\left(\operatorname{Syz}_{1}^{\widetilde{S}}(\widetilde{I})\right)-(d-1) \geq \operatorname{maxdeg}\left(\operatorname{Syz}_{1}^{S}(I)\right)-(d-1) .
$$

Example 4.2. We will apply Construction 4.1 to Koh's examples based on the Mayr-Meyer [MM] construction. For $r \geq 1$, Koh constructed in [Ko] an ideal $I_{r}$ generated by $22 r-3$ quadrics and one linear form in a polynomial ring with $22 r-1$ variables, and such that $\operatorname{maxdeg}\left(\operatorname{Syz}_{1}\left(I_{r}\right)\right) \geq 2^{2^{r-1}}$. The construction above produces an ideal $\widetilde{I}_{r}$ generated by $22 r-2$ quadrics in a polynomial ring with $22 r$ variables, and such that $\operatorname{maxdeg}\left(T_{r}\right) \geq 2^{2^{r-1}}-1$. On the other hand, by Theorem 4.3,

$$
\operatorname{deg}\left(T_{r}\right) \leq 2^{\min \{22 r-2,22 r\}}-1=2^{22 r-2}-1 .
$$

Thus, $\operatorname{deg}\left(T_{r}\right)<\operatorname{maxdeg}\left(T_{r}\right)$ for $r \geq 10$.

Now, we turn to Rees Algebras. 
Theorem 4.3. Let $M$ be an ideal generated by $m \geq 1$ forms of the same degree $d \geq 2$ in $R=k\left[X_{1}, \ldots, X_{n}\right]$, and $R_{M} \cong R[M t]$ be the Rees Algebra of $M$ which is considered as a standard graded quotient of the polynomial ring $R\left[Y_{1}, \ldots, Y_{m}\right]$. Then,

$$
\operatorname{deg}\left(R_{M}\right) \leq \frac{d^{\min \{m, n\}}-1}{d-1}
$$

and equality holds if further $M$ is $\left(X_{1}, \ldots, X_{n}\right)$-primary or its $m$ generators form a regular sequence.

Proof. Call $g_{1}, \ldots, g_{m}$ the given $m$ generators of $M$.

First give bidegree $(1,0)$ to the $X_{i}$ 's and bidegree $(0,1)$ to the $Y_{i}$ 's.

Notice that each bigraded component $\left(R_{M}\right)_{p, j}$ for this bigrading has at most a vector space dimension equal to the one of the Rees algebra associated to generic forms (ones with indeterminate coefficients). Indeed, this dimension can be computed as the rank of a Sylvester matrix associated to the collection of elements $g_{i_{1}} \cdots g_{i_{j}}$ with $i_{1} \leq \cdots \leq i_{j}$ in the degree $p+j d$.

This inequality in turn shows that the Hilbert function of $R_{M}$ is bounded above by the one given by generic forms (over the extension of $k$ generated by the coefficients).

If $m \leq n$ the generic forms are providing a regular sequence. And any complete intersection has its Rees algebra resolved by the Eagon-Northcott complex of the $(2 \times m)$-matrix with maximal minors $Y_{s} g_{r}-Y_{r} g_{s}$.

Let $\operatorname{Hilb}_{R_{M}}(u)$ be the Hilbert series of $R_{M}$. Whenever $m \leq n$ this shows the (term by term) inequality :

$$
\operatorname{Hilb}_{R_{M}}(u) \leq \frac{\left(1-u^{d}\right)^{m-1}+u^{d}\left(\sum_{i=0}^{m-2}\left(1-u^{d}\right)^{i}(1-u)^{m-i-1}\right)}{(1-u)^{m+n}} .
$$

As $M \neq 0$, the dimension of the Rees algebra of $M$ is the same as the one of a complete intersection and hence the inequality above shows that the degree of $R_{M}$ is bounded above by the one corresponding to a complete intersection, whose value is $d^{m-1}+d^{m-2}+\cdots+d+1$ by the above formula for the complete intersection case.

Notice further that any graded ideal generated in degree $d$ is a subideal of $J:=$ $\left(X_{1}, \ldots, X_{n}\right)^{d}$. It follows that the Hilbert series of $R_{M}$ is bounded above by the one of $R_{J}$. Again, recall that $R_{M}$ and $R_{J}$ have same dimension, hence

$$
\operatorname{deg}\left(R_{M}\right) \leq \operatorname{deg}\left(R_{J}\right)=\frac{d^{n}-1}{d-1}
$$

We computed the multiplicity in the complete intersection case, and if $M$ is $\left(X_{1}, \ldots, X_{n}\right)$ primary, it contains an ideal $M^{\prime}$ generated by a regular sequence of forms of degree $d$. The degree of $R_{M}$ is hence bounded below by the degree of $R_{M^{\prime}}$ and above by the one $R_{J}$; these are both equal to $\frac{d^{n}-1}{d-1}$ and the conclusion follows. 


\section{Regularity is bounded in terms of multiplicity}

In this section we show that an upper bound on regularity of non-degenerate prime ideals in terms of the multiplicity alone follows from the recent work of Ananyan and Hochster $[\mathrm{AH}]$, who solved Stillman's Conjecture. From now on, the polynomial rings occurring in the paper are standard graded.

Lemma 5.1. Let $L_{1}$ and $L_{2}$ be two homogeneous ideals of $U=k\left[X_{1}, \ldots, X_{N}\right]$ whose number and degrees of generators are bounded by a constant $c$. Then the number and the degrees of the generators of $L_{1} \cap L_{2}$ are bounded by a constant depending only on c.

Proof. Looking at the exact sequence

$$
0 \longrightarrow \frac{U}{L_{1} \cap L_{2}} \longrightarrow \frac{U}{L_{1}} \oplus \frac{U}{L_{2}} \longrightarrow \frac{U}{L_{1}+L_{2}} \longrightarrow 0
$$

we infer the following inequality for any $j \in \mathbb{Z}$ :

$$
\begin{aligned}
\operatorname{dim}_{k} \operatorname{Tor}_{0}^{U}\left(\frac{U}{L_{1} \cap L_{2}}, k\right)_{j} \leq & \operatorname{dim}_{k} \operatorname{Tor}_{0}^{U}\left(\frac{U}{L_{1}}, k\right)_{j}+\operatorname{dim}_{k} \operatorname{Tor}_{0}^{U}\left(\frac{U}{L_{2}}, k\right)_{j} \\
& +\operatorname{dim}_{k} \operatorname{Tor}_{1}^{U}\left(\frac{U}{L_{1}+L_{2}}, k\right)_{j} .
\end{aligned}
$$

By $\left[\mathrm{AH}\right.$, Theorem D (a)] the regularity and the graded Betti numbers of $\frac{U}{L_{1}+L_{2}}$ are bounded by a constant depending only on $c$, so we get the desired property.

Theorem 5.2. Let $e$ and $h$ be positive integers and $k$ be a field. There exist constants, depending only on $e$ and $h$, bounding respectively the projective dimension, regularity, and the graded Betti numbers of every homogeneous unmixed radical ideal of multiplicity e and height $h$ in a standard graded polynomial ring over $k$.

Proof. We may assume $e \geq 2$. Let $L$ be a homogeneous unmixed radical ideal of $U=$ $k\left[X_{1}, \ldots, X_{N}\right]$ of multiplicity $e$ and height $h$.

First, we will show that the ideal $L$ contains a regular sequence $g_{1}, \ldots, g_{h}$ of forms of degrees less than or equal to $e$. Choose a Noether normalization $k\left[X_{1}, \ldots, X_{d}\right]$ (this may need a finite extension of the base field to change coordinates if $|k| \leq e$, but this extension keeps $L$ unmixed and radical and does not affect the invariants we are bounding). Then the generators of $L \cap k\left[X_{1}, \ldots, X_{d}, X_{d+i}\right]=\left(g_{i}\right)$ for $i=1, \ldots h$ form a regular sequence of forms of degrees at most $e$.

Let $\mathfrak{b}:=\left(g_{1}, \ldots, g_{h}\right)$ and

$$
m:=\sum_{i=1}^{h}\left(\operatorname{deg} g_{i}-1\right)=\operatorname{reg}(U / \mathfrak{b}) .
$$

If $L=\mathfrak{b}$ the assertion is clear, so suppose $L \neq \mathfrak{b}$. If $\mathfrak{p}_{i}$ is a minimal prime of $\mathfrak{b}$, then there exists a form $f_{i}$ of degree $m$ such that $\mathfrak{p}_{i}=\mathfrak{b}:\left(f_{i}\right)$ (by $[\mathrm{Ch} 3,4.1]$ or $[\mathrm{CU}, 1.2]$, for instance). 
Hence, if $L=\cap_{i=1}^{a} \mathfrak{p}_{i}$, then $L=\mathfrak{b}:(f)$ with $f:=\sum_{i=1}^{a} f_{i}$. The exact sequence

$$
0 \longrightarrow U / L(-m) \longrightarrow U / \mathfrak{b} \longrightarrow U /(\mathfrak{b}+(f)) \longrightarrow 0
$$

then shows that $\operatorname{reg}(U / L)=\operatorname{reg}(U /(\mathfrak{b}+(f)))-m+1$. Hence the regularity of $L$ is bounded by the one of an ideal generated in degrees $(e, \ldots, e, m)$ such that $e$ is repeated $h$ times, and $m \leq h(e-1)$.

By $[\mathrm{AH}$, Theorem D (a)] it follows that the projective dimension, regularity, and Betti numbers of $L$ are bounded as well by constants depending only on $e$ and $h$.

Corollary 5.3. Let e be a positive integer and $k$ be an algebraically closed field. There exist constants, depending only on e, bounding the projective dimension, regularity and graded Betti numbers of every homogeneous non-degenerate prime ideal in a polynomial ring over $k$ of multiplicity $e$.

Proof. The claim follows immediately by Theorem 5.2 since the height of a homogeneous non-degenerate prime ideal in a polynomial ring over an algebraically closed field is less than its multiplicity.

This corollary also holds for reduced, equidimensionnal ideals that are connected in codimension 1 (or connected in codimension $r$, for any given $r \geq 1$ ). Also the conclusion concerning the regularity holds without the condition of being non-degenerate.

Examples 5.4. We give three examples. They show that Corollary 5.3 cannot be generalized to radical ideals, nor to primary ideals.

(1) Fix $n \in \mathbb{N}$ and let

$$
M=\left(x_{1}, \ldots, x_{n}\right) \cap\left(y_{1}, \ldots, y_{n}\right) \subseteq U=k\left[x_{i}, y_{i} \mid i=1, \ldots, n\right] .
$$

Then $M$ is a non-degenerate unmixed radical ideal of multiplicity 2 , but

$$
\operatorname{projdim} U / M=2 n-1 \text {. }
$$

(2) Fix $n \in \mathbb{N}$ and let

$$
M=\left(x^{2}, x y, y^{2}, x a^{n}+y b^{n}\right) \subseteq U=k[x, y, a, b] .
$$

It follows from [En, Lemma 10] that

(i) $M$ is a non-degenerate ideal of regularity equal to $n+1$;

(ii) $e(U / M)=2$;

(iii) $M$ is $(x, y)$-primary.

(3) Fix $n, e \in \mathbb{N}$ with $e \geq 3$. By [HMMS, Theorem 1.2] there exists an ideal $M$ in a polynomial ring $U$ over $k$ such that:

(i) $M$ is a non-degenerate ideal of projective dimension at least $n$;

(ii) $e(U / M)=e$;

(iii) $M$ is $(x, y)$-primary, where $x$ and $y$ are independent linear forms. 
These examples still leave open the question whether there exists a bound on the regularity of unmixed radical ideals (over an algebraically closed field) in terms of the multiplicity alone.

Remarks 5.5. We provide three upper bounds on regularity. Use the notation in the introduction.

(1) According to a lemma which Mumford attributes to Castelnuovo [Mu, Lemma, p.101] and a lemma which appears in his joint work with Bayer [BM], one has for any graded ideal $L$ a procedure to estimate the regularity of $U / L$ by induction on the dimension.

If $k$ is infinite (one can reduce to this case), choose a general linear form $\ell$ and set $r:=\operatorname{reg}(U / L+(\ell))$ and $r^{\prime}:=\operatorname{reg}\left(U /(L+(l))^{s a t}\right)$. Then

(i) $\operatorname{reg}(U / L) \leq r+\operatorname{dim}_{k}\left(H_{\mathfrak{m}}^{0}(U / L)_{r}\right)$ if $\operatorname{dim}(U / L) \geq 1$, with $H_{\mathfrak{m}}^{0}(U / L)_{r}=\left(L^{s a t} / L\right)_{r} \subset(U / L)_{r}$.

(ii) $\operatorname{reg}\left(U / L^{\text {sat }}\right) \leq r^{\prime}+\operatorname{dim}_{k}\left(H_{\mathfrak{m}}^{1}(U / L)_{r^{\prime}}\right)$, if $\operatorname{dim}(U / L) \geq 2$, with $\operatorname{dim}_{k} H_{\mathfrak{m}}^{1}(U / L)_{r^{\prime}}=\operatorname{dim}_{k} H^{0}\left(X, \mathcal{O}_{X}\left(r^{\prime}\right)\right)-\operatorname{dim}_{k}\left(U / L^{\text {sat }}\right)$, where $X:=\operatorname{proj}(U / L)$.

Estimates are precise and easy to get whenever $\operatorname{dim}(U / L) \leq 1$. Item (i) gives an estimate of the form $(2 d)^{(n-1) !}$ for the regularity of an ideal generated in degrees at most $d$ in a polynomial ring in $n$ variables. Introducing (implicitely) an invariant measuring both the regularity and the defect of saturation, Caviglia-Sbarra [CS] proved a refinement of this estimate, reducing the exponent of $2 d$ to $2^{n-1}$ or close to $2^{\operatorname{dim}(U / L)}$.

To use (ii), one notices that whenever $X$ is reduced, then $\operatorname{proj}(U / L+(\ell))$ is reduced as well, and if the $X_{i}$ 's for $i=1, \ldots, s$ are the irreducible components of $X$, then

$$
\operatorname{dim}_{k} H^{0}\left(X, \mathcal{O}_{X}(\mu)\right) \leq \sum_{i} \operatorname{dim}_{k} H^{0}\left(X, \mathcal{O}_{X_{i}}(\mu)\right)
$$

and

for $\mu \geq 0$.

$$
\operatorname{dim}_{k} H^{0}\left(X, \mathcal{O}_{X_{i}}(\mu)\right) \leq\left(\begin{array}{c}
\mu+\operatorname{dim} X_{i} \\
\operatorname{dim} X_{i}
\end{array}\right) e\left(X_{i}\right)
$$

This last estimate is valid for any symbolic power of a prime ideal (i.e., for the unmixed part of the scheme defined by a power of a prime ideal). If $L$ is a homogeneous radical ideal or, more generally, an intersection of symbolic powers of prime ideals and $e$ is the sum of their multiplicities, it implies

$$
\operatorname{reg}(L) \leq(e+1)^{(\operatorname{dim}(U / L)) !} .
$$

The existence of a bound in these terms traces back to work of Kleiman (see Rossi-VallaTrung [RTV, Section 3]).

(2) It follows from results of Lazarsfeld that, in characteristic zero, if an homogeneous equidimensionnal ideal $L$ of height $h$ is defined in degree at most $d$, then there exists another ideal $I$ with same radical such that $L$ and $I$ coincide locally at primes $\mathfrak{p}$ such that $(U / L)_{\mathfrak{p}}$ is regular and $\operatorname{reg}(U / I) \leq h(d-1)$. See Chardin-D'Cruz [CD] for a more precise statement. 
As a consequence $\operatorname{reg}\left(U / L^{\text {sat }}\right) \leq h(d-1)$ if the projective scheme defined by $L$ has at most isolated singularities.

(3) Assume $X \subseteq \mathbb{P}^{n}$ is reduced and equidimensionnal of dimension $d$ over an algebraically closed field and $c_{j}$ is the number of connected components of scheme $X(j):=$ $X \cap H_{1} \cap \cdots \cap H_{j}$ for $j=0, \cdots, d:=\operatorname{dim} X$ and general hyperplanes $H_{i}$. Then $c_{0} \leq c_{1} \leq$ $\cdots \leq c_{d}=\operatorname{deg}(X)$ and $X(d)$ is not contained in any linear space of dimension $<n-\sigma$

with $\sigma:=\sum_{j=0}^{d-1} c_{j}$. Thus $\operatorname{deg}(X) \geq n-\sigma$. Also $c_{d-1}$ is bounded above by the number $s$ of irreducible components of $X$. If $X$ is the union of irreducible components $X_{i}$, we thus have

$$
\sum_{i=1}^{s} \operatorname{deg}\left(X_{i}\right)+\sum_{j=0}^{d-1}\left(c_{j}-1\right) \geq \operatorname{codim}(X)
$$

It follows that if $X$ is connected in codimension $r$, then

$$
\operatorname{codim}(X) \leq \operatorname{deg}(X)+(r-1)(s-1) \leq r \operatorname{deg}(X)-r+1 .
$$

\section{Prime ideals from designer ideals}

In this section, we apply the method in $[\mathrm{MP}]$ to the designer ideals constructed by Ullery in [Ul].

First we define notation related to maximal shifts. Let $T=k\left[x_{1}, \ldots, x_{n}\right]$ and let $M$ be a finitely generated $T$-module. Set

$$
t_{i}^{T}(M)=\max \left\{j \mid \operatorname{Tor}_{i}^{T}(M, k)_{j} \neq 0\right\}=\max \left\{j \mid \beta_{i j}^{T}(M) \neq 0\right\} .
$$

Thus $t_{0}^{T}(M)$ is the maximal degree of an element in a minimal generating set of $M$ and $t_{1}^{T}(M)$ is the maximal degree of a minimal first syzygy of $M$. The maximal shifts $t_{i}^{T}(M)$ are related to regularity by

$$
\operatorname{reg}(M)=\max _{0 \leq i \leq \operatorname{projdim}(M)}\left\{t_{i}^{T}(M)-i\right\} .
$$

We state a version of a result of Ullery:

Theorem 6.1 ([Ul, Theorem 1.3]). Let $T=k\left[x_{1}, \ldots, x_{n}\right]$ and let $M$ be a finitely generated $T$-module generated in a single non-negative degree with strictly increasing sequence of maximal graded shifts $\left(t_{0}^{T}(M), t_{1}^{T}(M), \ldots, t_{r}^{T}(M)\right)$. Set $a=t_{0}^{T}(M)$ and fix a positive integer $N$ such that the number of elements in a minimal homogeneous generating set of $M$ is $\leq\left(\begin{array}{c}N+a-1 \\ a\end{array}\right)$. Then there exists an ideal $J_{M}$ in $S=T\left[y_{1}, \ldots, y_{N}\right]$ such that

$$
t_{i}^{S}\left(J_{M}\right)= \begin{cases}t_{i+1}^{T}(M) & \text { if } 0 \leq i \leq r-1 \\ t_{r}^{T}(M)+i-r+1 & \text { if } r \leq i \leq N+r-1 .\end{cases}
$$

In particular, if we pick $M$ to be generated in degree $a=1$, and $N$ sufficiently large, then there exists an ideal $J_{M}$ generated by homogeneous quadrics with any strictly increasing sequence as an initial sequence of its maximal graded shifts. 
We now take the step-by-step homogenization of the defining prime ideal of the Reeslike algebra of $J_{M}$ to produce prime ideals over any field with generators in degree at most 6 and arbitrarily large degree of first syzygies:

Theorem 6.2. Fix a positive integer $s \geq 9$ and field $k$. There exists a non-degenerate prime ideal $P$ in a polynomial ring $R$ over $k$ with $t_{0}^{R}(P)=6$ and $t_{1}^{R}(P)=s$.

Proof. Take $T=k\left[x_{1}, x_{2}, x_{3}\right]$ and $M=\operatorname{Ext}_{S}^{3}\left(T /\left(x_{1}, x_{2}, x_{3}\right)^{s-3}, S\right)(4-s)$ (where $(4-s)$ denotes a shift of degrees). Then $M$ is a Cohen-Macaulay module with Betti table of the form:

\begin{tabular}{r|cccc} 
& 0 & 1 & 2 & 3 \\
\hline $1:$ & $*$ & $*$ & $*$ & - \\
$2:$ & - & - & - & - \\
$3:$ & - & - & - & - \\
$\vdots$ & - & - & - & - \\
S-4: & - & - & - & $*$
\end{tabular}

where "*" denotes a non-zero entry and "-" denotes a zero entry. Thus,

$$
t_{0}^{T}(M)=1, t_{1}^{T}(M)=2, t_{2}^{T}(M)=3, t_{4}^{T}(M)=s-1 .
$$

By Theorem 6.1, there is an ideal $J$ in a larger polynomial ring $S$ with

$$
t_{0}^{S}(J)=2, t_{1}^{S}(J)=3, t_{2}^{S}(J)=s-1
$$

and $t_{i}^{S}(J)=s+i-3$ for $3 \leq i \leq \operatorname{projdim} J$.

Now let $P$ be the step-by-step homogenization of the defining prime ideal of the Reeslike algebra of $J$ in a larger polynomial ring $R$, as constructed in [MP]. By [MP, Theorem $1.6]$,

$$
t_{0}^{R}(P)=\max \left\{2\left(t_{0}^{S}(J)+1\right), t_{1}^{S}(J)+1\right\}=\max \{6,4\}=6 .
$$

The structure of the minimal free resolution of $P$ in [MP, Theorem 3.10] implies that

$$
t_{1}^{R}(P)=\max \left\{3\left(t_{0}^{S}(J)+1\right), t_{1}^{S}(J)+t_{0}^{S}(J)+2, t_{2}^{S}(J)+1\right\}=\max \{9,7, s\}=s .
$$

\section{Ideals with Large Regularity}

In this section we provide an infinite family of counterexamples to the Eisenbud-Goto Regularity Conjecture that do not rely on the Mayr-Meyer construction.

Proposition 7.1. Let $T$ be a polynomial ring over a field $k$ and let $J=(f, g, h)$ be a homogeneous ideal of $T$ such that $f, g, h$ all have the same degree. Let $x, y$ be new variables and set $S=T[x, y]$. Let

$$
I=\left(x^{3}, y^{3}, x^{2} f+x y g+y^{2} h\right) .
$$


Then

$$
\operatorname{reg}_{S}(S / I) \geq \operatorname{reg}_{T}(T / J)+4 .
$$

Proof. Note that $S / I$ is finitely generated as a $T$-module. In fact, $x^{2} y^{2}(S / I) \cong(T / J)(-4)$ is a $T$-direct summand of $S / I$. Since $S$ is faithfully flat over $T$, we have

$$
\operatorname{reg}_{S}(S / I) \geq \operatorname{reg}_{T}\left(x^{2} y^{2}(S / I)\right)=\operatorname{reg}_{T}(T / J)+4 .
$$

In [Ca, Example 4.2.1] Caviglia showed that if $T=k\left[z_{1}, z_{2}, z_{3}, z_{4}\right]$ and

$$
J=\left(z_{1}^{d}, z_{2}^{d}, z_{1} z_{3}^{d-1}-z_{2} z_{4}^{d-1}\right)
$$

with $d \geq 2$, then $\operatorname{reg}(T / J)=d^{2}-2$. We set $S=T[x, y]$ and

$$
I=\left(x^{3}, y^{3}, x^{2} z_{1}^{d}+x y\left(z_{1} z_{3}^{d-1}-z_{2} z_{4}^{d-1}\right)+y^{2} z_{2}^{d}\right) .
$$

By the previous proposition we see that $\operatorname{reg}(S / I) \geq d^{2}+2$ for $d \geq 2$, while the degrees of the three generators of $I$ are 3,3, and $d+2$. By [MP, Theorem 1.6] we obtain the following result:

Theorem 7.2. Let $P$ be the step-by-step homogenization of the Rees-like algebra of the ideal $I$ above, in the polynomial ring $R$ (as constructed in $[\mathrm{MP}]$ ). Then

$$
\begin{aligned}
& \operatorname{deg}(R / P)=32(d+3) \\
& \operatorname{reg}(R / P) \geq d^{2}+d+12 .
\end{aligned}
$$

In particular, the Eisenbud-Goto conjecture fails when $d \geq 34$.

Acknowledgements. We are very grateful to David Eisenbud for useful discussions.

\section{References}

[AH] T. Ananyan, M. Hochster, Small Subalgebras of Polynomial Rings and Stillman's Conjecture arXiv:1610.09268.

[BM] D. Bayer and D. Mumford: What can be computed in Algebraic Geometry?, Computational Algebraic Geometry and Commutative Algebra, Symposia Mathematica, Volume XXXIV, Cambridge University Press, Cambridge, 1993, 1-48.

[BS] D. Bayer and M. Stillman: On the complexity of computing syzygies. Computational aspects of commutative algebra, J. Symbolic Comput. 6 (1988), 135-147.

[BI] W. Bruns and B. Ichim: On the coefficients of quasipolynomials, Proc. Amer. Soc. 135 (2006), 1305-1308.

[Ca] G. Caviglia: Koszul Algebras, Castelnuovo-Mumford Regularity, and Generic initial ideals, $\mathrm{PhD}$ Thesis. University of Kansas (2005).

[CS] G. Caviglia and E. Sbarra: Characteristic-free bounds for the Castelnuovo-Mumford regularity, Compos. Math. 141 (2005), 1365-1373.

[Ch] M. Chardin: Some results and questions on Castelnuovo-Mumford regularity, Syzygies and Hilbert functions, Lect. Notes Pure Appl. Math. 254, Chapman \& Hall/CRC, Boca Raton, FL, 2007. 
[Ch2] M. Chardin: Bounds for Castelnuovo-Mumford regularity in terms of degrees of defining equations, Commutative algebra, singularities and computer algebra (Sinaia, 2002), 67-73, NATO Sci. Ser. II Math. Phys. Chem., 115, Kluwer Acad. Publ., Dordrecht, 2003.

[Ch3] M. Chardin: Applications of some properties of the canonical module in computational projective algebraic geometry, Symbolic computation in algebra, analysis, and geometry (Berkeley, CA, 1998), J. Symbolic Comput. 29 (2000), 527-544.

[CD] M. Chardin, C. D'Cruz: Castelnuovo-Mumford regularity: examples of curves and surfaces, J. Algebra 270 (2003), 347-360.

[CFN] M. Chardin, A. Fall, U. Nagel: Bounds for the Castelnuovo-Mumford regularity of modules, Math. Z. 258 (2008), 69-80.

[CU] M. Chardin, B. Ulrich: Liaison and Castelnuovo-Mumford regularity, Amer. J. Math. 124 (2002), 1103-1124.

[DLL] Jan Draisma, Michal Lason, Anton Leykin: Stillman's conjecture via generic initial ideals, arXiv:1802.10139.

[EG] D. Eisenbud, S. Goto Linear free resolutions and minimal multiplicity, J. Algebra 88 (1984), 89133.

[EHV] D. Eisenbud, C. Huneke, W. Vasconcelos, Direct methods for primary decomposition, Inventiones Mathematicae, 110 (1992), 207-235.

[En] B. Engheta: On the projective dimension and the unmixed part of three cubics, J. Algebra 316 (2007), 715-734.

[ESS] Daniel Erman, Steven V Sam, Andrew Snowden: Big polynomial rings and Stillman's conjecture, arXiv:1801.09852.

[Ga] A. Galligo: Théorème de division et stabilité en géométrie analytique locale, Ann. Inst. Fourier (Grenoble) 29 (1979), 107-184.

[GLP] L. Gruson, R. Lazarsfeld, and C. Peskine: On a theorem of Castelnuovo and the equations defining projective varieties, Invent. Math. 72 (1983), 491-506.

[Gi] M. Giusti: Some effectivity problems in polynomial ideal theory, in Eurosam 84, Lecture Notes in Computer Science, 174, Springer (1984), 159-171.

[Hu] C. Huneke: On the symmetric and Rees algebra of an ideal generated by a d-sequence, J. Algebra 62 (1980), 268-275.

[HMMS] C. Huneke, P. Mantero, J. McCullough, A. Seceleanu: Multiple structures with arbitrarily large projective dimension supported on linear spaces, J. Algebra 447 (2016), 183-205.

[Ko] J. Koh: Ideals generated by quadrics exhibiting double exponential degrees, J. Algebra 200 (1998), 225-245.

[KPU] A. Kustin, C. Polini, B. Ulrich: Rational normal scrolls and the defining equations of Rees algebras, J. Reine Angew. Math. 650 (2011), 23-65.

[MM] E. Mayr and A. Meyer: The complexity of the word problem for commutative semigroups and polynomial ideals, Adv. in Math. 46 (1982), 305-329.

[MP] J. McCullough, I. Peeva: Counterexamples to the Eisenbud-Goto Regularity Conjecture, submitted.

$[\mathrm{Mu}]$ D. Mumford: Lectures on curves on an algebraic surface, With a section by G. M. Bergman, Annals of Mathematics Studies 59 Princeton University Press, Princeton, N.J., 1966.

[RTV] M. E. Rossi, N. V. Trung, G. Valla: Castelnuovo-Mumford regularity and finiteness of Hilbert functions, Commutative algebra, Lect. Notes Pure Appl. Math. 244 (2006), Chapman \& Hall/CRC, Boca Raton, FL, 193-209.

[Ul] B. Ullery: Designer ideals with high Castelnuovo-Mumford regularity, Math. Res. Lett. 21 (2014), 1215-1225. 
Department of Mathematics, Purdue U. 150 N. University Street, W. Lafayette, IN 47907 2067, USA

E-mail address: gcavigli@purdue.edu

Institut de mathématiques de Jussieu, UPMC, 4, Place Jussieu, F-75005 Paris, France

E-mail address: marc.chardin@imj-prg.fr

Mathematics Department, Iowa State University, 411 Morrill Road, Ames, IA 50011, USA

E-mail address: jmccullo@iastate.edu

Mathematics Department, Cornell University, IthacA, NY 14853, USA

Dipartimento di Matematica, Università degli Studi di Genova, Via Dodecaneso 35, 16146 , ITALY

E-mail address: varbaro@dima.unige.it 